\title{
Idiosyncratic Pain Patterns During Exhaustive Exercise
}

\author{
Agnė Slapšinskaité $\dot{1}^{1,2,3}$, Selen Razon $^{4}$, Natàlia Balagué $^{1}$, Arūnas Ščiupokas ${ }^{5}$, \\ Robert Hristovski $^{6}$ \& Gershon Tenenbaum ${ }^{7}$ \\ ${ }^{1}$ Complex Systems in Sport Research Group, Institut Nacional d'Educació Física de Catalunya (INEFC), \\ Universitat de Barcelona (UB). Avda. de l'Estadi, 12-22, 08038 Barcelona, Spain \\ ${ }^{2}$ Institute of Sports, Lithuanian University of Health Sciences (LUHS), Tilžès st. 18, LT - 47181, Kaunas, \\ Lithuania \\ ${ }^{3}$ Health Research Institute, LUHS, Tilžès st. 18, LT - 47181, Kaunas, Lithuania \\ ${ }^{4}$ Department of Kinesiology, West Chester University, 700 S. High St, West Chester, PA 19382, USA \\ ${ }^{5}$ Department of Neurology, Lithuanian University of Health Sciences, Eivenių g. 2, LT-50009 Kaunas, Lithuania \\ ${ }^{6}$ Department of Physical Education, Sport and Health, Ss. Cyril and Methodius University, Zeleznicka BB, 1000 \\ Skopje, Macedonia \\ ${ }^{7}$ Department of Educational Psychology and Learning Systems, Florida State University, 600 W. College Avenue, \\ Tallahassee, FL 32306, USA \\ Correspondence: Agnė Slapšinskaite, Complex Systems in Sport Research Group, Institut Nacional d'Educació \\ Física de Catalunya (INEFC), Universitat de Barcelona (UB). Avda. de l'Estadi, 12-22, 08038 Barcelona, Spain. \\ Tel: 34-93-425-5445; Fax: 34- 93-426-3617. E-mail: agneslapsinskaite@gmail.com
}

Received: July 5, 2017 Accepted: August 7, 2017 Online Published: April 27, 2018

doi:10.5539/gjhs.v10n6p44 URL: https://doi.org/10.5539/gjhs.v10n6p44

\begin{abstract}
The purpose of this study was to investigate the dynamical distribution of pain in constant cycling and running tasks up to the point of exhaustion. Ten participants $(\mathrm{M}=20.8$ years old, $\mathrm{SD}= \pm 1.03)$ ran and cycled at a "hard" intensity level (e.g., Borg's RPE $(6-20)=15)$. During task performance, participants reported their pain on a body map every $15 \mathrm{~s}$. Three distinct and consistent pain distribution patterns emerged: adders who added pain locations, jumpers who switched among pain locations, and adders-jumpers who both added and switched among pain locations throughout the effort. These distribution patterns had a significant effect $(p<.001)$ on pain stability (i.e., the time spent within the same pain location) and on total number of changes in pain locations $(p<0.04)$; which differed between the adders and jumpers $(\mathrm{p}<.035)$. Task endurance was associated with the total number of changes of pain locations $(r=.46, \mathrm{p}<.04)$. Finally, a significant effect of time on the number of symmetric locations $\chi^{2}(10,4)=16.17, \mathrm{p}<.003$ emerged in running. Idiosyncratic pain distribution patterns with more switching among pain locations throughout effort seemed to increase time on task. Further scientific evidence is needed for confirming the extent to which idiosyncratic pain distribution patterns account for and/or help pain management within clinical settings.
\end{abstract}

Keywords: pain locations, pain dynamics, idiosyncratic patterns, cycling, running, endurance, pain management.

\section{Introduction}

In the course of endurance activities, people experience exertive pain (Razon et al., 2010) or exercise-related discomfort. Exertive pain is associated with aversive sensations emanating mainly from the legs, respiratory system, proprioceptive systems, head, and abdomen (Tenenbaum et al., 1999). The term perceived exertion (PE) typically represents a mix of painful sensations, heaviness of breathing during exercise, and fatigue which results from the physical effort. However, researhcers have called for the study of exertive pain as a distinct perceptual marker (Dannecker \& Koltyn, 2014; Haile, Gallagher \& Robertson, 2015). To that end, exertive pain during exercise has been measured via pain intensity scales (Cook, O'Connor, Oliver, \& Lee, 1998), numeric rating scales, visual analog scales (Tripathi \& Kumar, 2014), as well as pain mapping procedures for identifying painful locations (Margolis, Tait, , \& Krause, 1986; Slapsinskaite, Hristovski, Razon, Balagué, \& Tenenbaum, 2017; Slapsinskaite, Razon, Balagué Serre, Hristovski, \& Tenenbaum, 2015).

A taxonomy for complex spatial pain patterns was developed following multi-site pain identifications, which 
revealed 475 spatial pain patterns, grouped into seven categories (Schmidt \& Baymeister, 2007). This method emphasizes the importance of spatial patterns, not only for quantifying the painful body sites as an indicator of pain severity (Graven-Nielsen \& Arendt-Nielsen, 2010), but also for observing the distribution of pain for each person. As a result, further investigation of both static and dynamic pain patterns were warranted (Tarr \& Thomas, 2011). Indeed, defining idiosyncratic pain distribution patterns during exercise can help in the operational definition of perceived pain dynamics that can otherwise be missed by use of other approaches.

Delineating idiosyncratic pain distribution patterns during exercise can prove beneficial in that exertive and clinical pain can at least partly share common mechanisms. In exercise settings pain typically occurs in diverse muscle groups (Dannecker \& Koltyn, 2014). Somewhat similarly, in clinical settings the experience of multisite pain is common (Leveille, Bean, Ngo, McMullen, \& Guralnik, 2007). In fact, in clinical contexts, persistent musculoskeletal pain remains an essential feature of chronic pain syndromes.

Of specific interest herein, perception of pain typically requires attentional allocation. On the other hand, the perception of pain requires a short-term integration between a number of continuously interplaying components such as environment, periphery and the brain itself. Moreover, perception of pain and attention to pain per se are multidimensional and tend to interrelate through hierarchical dynamical process (Rabinovich, Tristan, \& Varona, 2015). Spontaneous attentional fluctuations toward and "away from pain" and their neural mechanisms can be termed "perception decoupling" or "disengagement of attention" from perception (Schooler et al., 2011). Recently, it was demonstrated how dynamics of mental hierarchies may be formed by component perceptions (i.e., pain) that dwell over different time scales (Slapsinskaite et al., 2017). This is a finding that demonstrates that the phenomenological subjective experience, so called qualia (Chalmers, 1996) are grounded in identical dynamical principles to the neural tissue, i.e., the brain.

The purpose of this study was to investigate the dynamical distribution of pain in constant cycling and running tasks up to the point of exhaustion. We hypothesized that instead of generalized patterns, idiosyncratic dynamics of exertive pain would emerge. Consequently, we expected that the results of this study would help advance the understanding of pain dynamics in effort settings. We also considered that due to the potentially common grounds shared between exercise-related pain distribution and medical pain distribution, these findings would contribute to the understanding of medical pain dynamics within clinical settings. Finally, of interest to applied realms, sensation of pain and exertion remain major antecedents for the cessation of applied effort and reduced performance (Kayser, 2003). Consequently, researchers have warranted further investigation of effort-induced pain (Astokorki \& Mauger, 2017). A closer understanding of pain dynamics within efforts settings could allow improved strategies to manage exertive pain and facilitate performance within effort settings (Chow \& Etnier, 2017).

\section{Method}

\subsection{Participants}

Sample size was determined using the $\mathrm{G}^{*}$ Power 3.1 program (Faul, Erdfelder, Lang, \& Buchner, 2007). Consistent with previous work (Balagué et al., 2015), 10 participants were needed for achieving a $\mathrm{d}=1.0, \alpha<.05$, and power of $(1-\beta) 72=.80$. Ten Caucasian students (four females and six males, Mage $=20.8$ years, $\mathrm{SE}= \pm 1.33$, age range 19-24 years) majoring in physical education were recruited. All the participants were healthy and injury-free with no counter indication for exercise testing. Participants were physically active and familiar with cycling and running at high intensities for extended durations.

\subsection{Intervention and Procedure}

Completion of this study took three sessions. Each of the sessions lasted approximately 30 minutes and they were separated by one-week intervals. At week 1, participants completed baseline incremental cycling and running tests to determine the workload and velocity values corresponding to their RPE (6-20 Borg's scale) $=15$ (i.e., heavy; see Borg, 1998). At this time, participants were familiarized with the use of body maps and reporting procedures (see familiarization). At the week 2 and 3 participants completed the constant-power cycling and constant velocity running tasks, respectively, in a counter-balanced assigned order. Prior to the onset of the study, participants completed a health history questionnaire, and an informed consent form. Clinical Research Ethics Committee of the Sports Administration of Catalonia has approved this study. All the experimental procedures associated with this study were conducted in a way that is consistent with the Helsinki Declaration.

\subsubsection{Monitoring for Pain}

Throughout the exercise protocol, every $15 \mathrm{~s}$, upon the researcher's prompt, participants reported body locations with discomfort and pain, using a body map (adapted from Margolis, Tait, \& Krause, 1986) (see Figure 1). The 
rational for selecting this reporting strategy was to provide ample data points and limiting potentially deleterious effects of simultaneously reporting somatic sensations and keeping an internal focus of attention (Villemure \& Bushnell, 2002). For the purposes of this assessment, participants' instructions included the following:

"When prompted, please report the locations of discomfort and pain (if you feel it, independently of its magnitude) using the numbers on the body map placed in front of you."
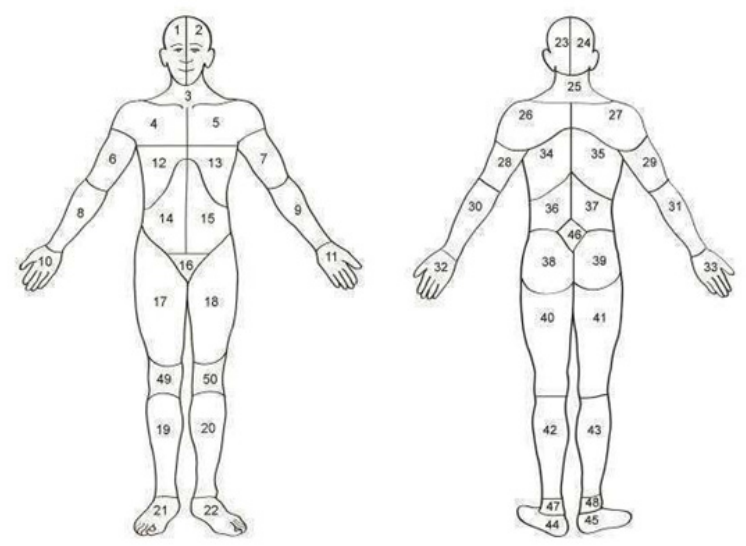

Figure 1. Body map

Note. Head (zone 1, 2, 23, or 24); neck (zone 3 or 25), shoulders (zone 4, 5, 26,27); arms (zone 6, 7, 8, 9, 28, 29, 30 or 31); hand (zones 10,11,32,33); ribs or chest (zone 12 or 13); abdomen (zone 14 or 15), back (zones 34, 35, 36, 37), buttocks or hips (zone 38 or 39); genitalia (zone16), legs (zone 17, 18, 19, 20, 40, 41, 42 or 43); feet (zone 21, 22, 44 or 45). Adapted from Margolis, Tait, \& Krause (1986).

\subsubsection{Familiarization and Baseline Tests}

A cycle ergometer (Sport Excalibur 925900) was used both for the baseline incremental cycling test and the constant-power cycling task. Cycle ergometer's saddle and handlebar were fitted to each participant's comfort. Following a $2 \mathrm{~min}$ rest period, participants were instructed to cycle at $70 \mathrm{rpm}$. Male and female participants began cycling with a load of $25 \mathrm{~W}$ and $20 \mathrm{~W}$ respectively. Participants' load was increased each minute by the same increment (i.e., $25 \mathrm{~W}$ for males, $20 \mathrm{~W}$ for females) until they reported RPE=15. At this point, participants kept cycling for $2 \mathrm{~min}$ and reported on the pain scales. Participants reported their RPE within the last $10 \mathrm{~s}$ of each workload increment. For each participant, his/her workload intensity coinciding the RPE $=15$ was noted.

The baseline incremental running test was performed on a treadmill (HP Cosmos treadmill). The running test started with a $2 \mathrm{~min}$ rest. Next, participants ran at an initial velocity of $5 \mathrm{~km} / \mathrm{h}$ with increases of $5 \mathrm{~km} / \mathrm{h}$ each minute (for females) and $1 \mathrm{~km} / \mathrm{h}$ each minute (for males) until RPE $=15$. At this intensity, participants kept running for 2 min and reported on the pain scales (see above). During the last $10 \mathrm{~s}$ of every imposed workload increment, participants reported their RPE. The workload values corresponding to RPE $=15$, were recorded for each participant.

\subsubsection{Cycling Task}

Cycling task included an incremental warm-up phase (identical to baseline protocol) and the constant-power cycling task. Cycling task was performed at constant power up to volitional exhaustion. Participants started the cycling task when they reported RPE $=15$ during the warm up phase. The task was terminated when they reached volitional exhaustion meaning they could no longer cycle at the preset cadence for five consecutive seconds while keeping a sitting position. For the purposes of the warm-up protocol, the RPE scale (6-20) was placed in front of the participants at eye level. During the cycling protocol, RPE scale was replaced by the body map. Analysis included data from participants who reported $\mathrm{RPE}=15$ at the same target workload during the baseline incremental warm-up phase.

\subsubsection{Running Task}

Running task included an incremental warm-up (identical to baseline protocol) and the running task. Running task 
was performed at constant velocity up to volitional exhaustion. Participants started the running task when they reported $\mathrm{RPE}=15$ during the warm up phase. The task was terminated when they reached volitional exhaustion meaning they could no longer maintain the required velocity while running. For the purposes of the warm-up protocol, the RPE scale (6-20) was placed in front of the participants at eye level. During the running protocol, RPE scale was replaced by the body map. Analysis included data from participants who reported RPE $=15$ at the same target velocity during the baseline incremental warm-up phase.

At the exception of the researcher's prompts for reporting pain locations, participants completed the tasks with no verbal communication. For cross validity purposes, both tasks were videotaped. At task completion, using a Likert-type scale of 11 point with anchors ranging from 0 (not at all) to 10 (greatly), participants responded to two items that gauged task-commitment: (a) "Have you pedaled/ran as long as you can, up to your exhaustion point?" and, commitment to the protocol (b) "Have you reported all the changes in your discomfort and pain locations?"

\subsection{Data Analysis}

Once recorded, all the locations for each participant were transformed to binary vectors, where 1 meant presence of pain, and 0 - no pain, to obtain the time series with topological configuration of the perceived pain regions while performing the constant cycling and running tasks. To determine the pain dynamic patterns, the binary vectors time series were analyzed with "and" and "or" logical segments.

Principal component analysis (PCA) was used to reduce the dimensionality of the local pain data (obtained from the 50-item pain map). The PCA method was used to extract the components and to obtain a maximal dimensional reduction of the data. The number of components to retain was determined through the number of salient PCs of the first order by applying Kaiser-Guttman criterion (eigenvalue $\lambda \geq 1$; Yeomans $\&$ Golder, 1982) and by the visual inspection of a scree-plot. The parsimonious PC structure was obtained by use of the normalized Varimax criterion.

A Wilcoxon matched pairs test was used to contrast between cycling and running conditions for total number of changes in pain locations and pain stability (i.e., the time spent within the same pain location). Effect of pain distribution patterns on the total number of changes in pain locations and pain stability were subjected to one-way analysis of variance (ANOVA) followed by Tukey's HSD tests for post hoc comparisons. To assess possible relations between the pain dynamics and task endurance, a Pearson correlation was computed.

Reported number of locations with pain while performing the tasks were plotted for each participant. The symmetric pain (i.e., axial distribution of the same locations in right and left sides of the body) were computed for each participant separately. Each time series was divided into five non-overlapping temporal windows (time to volitional exhaustion of the participant/5) for cycling and running tasks. The probability to experience symmetric pain was computed for each temporal window and took into account only the reported pain. A Friedman ANOVA was used to analyze the number of locations variance over time. Effect sizes (Cohen's d) were calculated to determine means' differences at $\mathrm{p}<.05$. Data analysis was completed using SPSS version 20.0 (SPSS Inc, Chicago, IL). The level of statistical significance was set at $\mathrm{p}<.05$.

\section{Results}

\subsection{Idiosyncratic Pain Distribution Patterns}

Three groups of participants were identified based on the distribution pattern of pain during cycling and running. Of the 10 participants, $20 \%$ reported an increasing amount of locations without losing any previously reported locations. These participants were termed adders as their reporting pattern matched the logic "and". Next $20 \%$ of the participants shifted in between reported locations without adding any new locations. These participants were termed jumpers since their reporting pattern matched the logic "or". Finally, $60 \%$ of the participants combined both patterns of reporting and they were termed adder-jumpers since their reporting pattern matched a logic of both "and" and "or".

\subsection{Principle Component Analysis (PCA) of Pain Data Series}

The Kaiser-Guttmann procedure, which relied on the pain distribution pattern, revealed 3 principle components (PCs) and 4 PCs in adders, 7 PCs and 9 PCs in jumpers, and 5 PCs and 6 PCs in adders-jumpers in cycling and running tasks, respectively. On average, adders accounted for $95.02 \%$ and $97.47 \%$ of the variance in cycling and in running, respectively. Jumpers accounted for $92.12 \%$ of the variance in cycling and $94.00 \%$ in running. Finally, adders-jumpers accounted for $95.09 \%$ of the variance in cycling and $96.96 \%$ of variance in running. On average, in the cycling task the first PC accounted for $62.02 \%, 49.92 \%$ and $63.98 \%$, of the total variance in adders, jumpers and adder-jumpers, respectively. In the course of the running task the first PC on average accounted for $64.02 \%$, 
$52.92 \%$ and $58.82 \%$, of the total variance in adders, jumpers and adder-jumpers, respectively. The remaining PCs contributed substantially less to the accounted variance (See Table 1). The accounted variances per configuration by the whole system of PCs (commonalities) in both task were $\mathrm{h} 2=.95 \pm .007$.

Table 1. Principal Components Analysis (PCA).

\begin{tabular}{|c|c|c|c|c|c|c|c|c|c|c|c|}
\hline \multicolumn{5}{|c|}{ Cycling } & \multirow[b]{3}{*}{$\mathrm{n}$} & \multicolumn{6}{|c|}{ Running } \\
\hline \multirow[b]{2}{*}{$\mathrm{PC}$} & \multicolumn{2}{|c|}{ Average } & \multicolumn{2}{|l|}{ SD } & & \multirow[b]{2}{*}{$\mathrm{PC}$} & \multicolumn{2}{|c|}{ Average } & \multicolumn{2}{|l|}{ SD } & \multirow[b]{2}{*}{$\mathrm{n}$} \\
\hline & $\bar{\lambda}$ & Cum \% Var & $\lambda$ & Cum \% Var & & & $\bar{\lambda}$ & Cum \% Var & $\bar{\lambda}$ & Cum \% Var & \\
\hline 1 & 28.15 & 58.64 & 11.96 & 14.05 & 11 & 1 & 20.76 & 57.59 & 7.11 & 11.33 & 11 \\
\hline 2 & 7.8 & 72.52 & 3.53 & 11.84 & 11 & 2 & 7.42 & 78.01 & 3.54 & 8.26 & 11 \\
\hline 3 & 1.53 & 85.5 & 2.19 & 10.15 & 11 & 3 & 3.89 & 88.14 & 2.31 & 7.2 & 11 \\
\hline 4 & 2.03 & 89.61 & 1.63 & 9.55 & 10 & 4 & 1.85 & 92.08 & 1.16 & 5.13 & 10 \\
\hline 5 & 1.75 & 92.57 & 1.12 & 9.18 & 8 & 5 & 1.24 & 95.71 & 0.97 & 4.32 & 9 \\
\hline 6 & 1.31 & 94.4 & 1.17 & 7.96 & 6 & 6 & 1.09 & 97.25 & 1.15 & 3.13 & 7 \\
\hline 7 & 0.61 & 99.98 & 0.09 & 0.03 & 2 & 7 & 0.76 & 97.71 & 0.67 & 2.14 & 5 \\
\hline \multirow[t]{5}{*}{8} & 0.03 & 100 & & & 1 & 8 & 0.69 & 98.47 & 0.49 & 1.27 & 4 \\
\hline & & & & & & 9 & 0.56 & 99.05 & 0.14 & 0.9 & 3 \\
\hline & & & & & & 10 & 0.32 & 99.63 & 0.22 & 0.64 & 3 \\
\hline & & & & & & 11 & 0.09 & 99.75 & & & 1 \\
\hline & & & & & & 12 & 0.03 & 100 & & & 1 \\
\hline
\end{tabular}

Note. Revealed principal components and accounted variance for the original pain data in cycling and running.

Figure 2 illustrates how different communities of painful zones emerged and decayed in time by the shifting projections on the extracted PCs. The dark regions represent high values of projections of zones on given PCs corresponding to larger projection values of vectors defining reported body pain sets. They represent formation of a correlated community of pain locations which is only temporarily stable. Each community eventually decayed to leave space for another community to form.

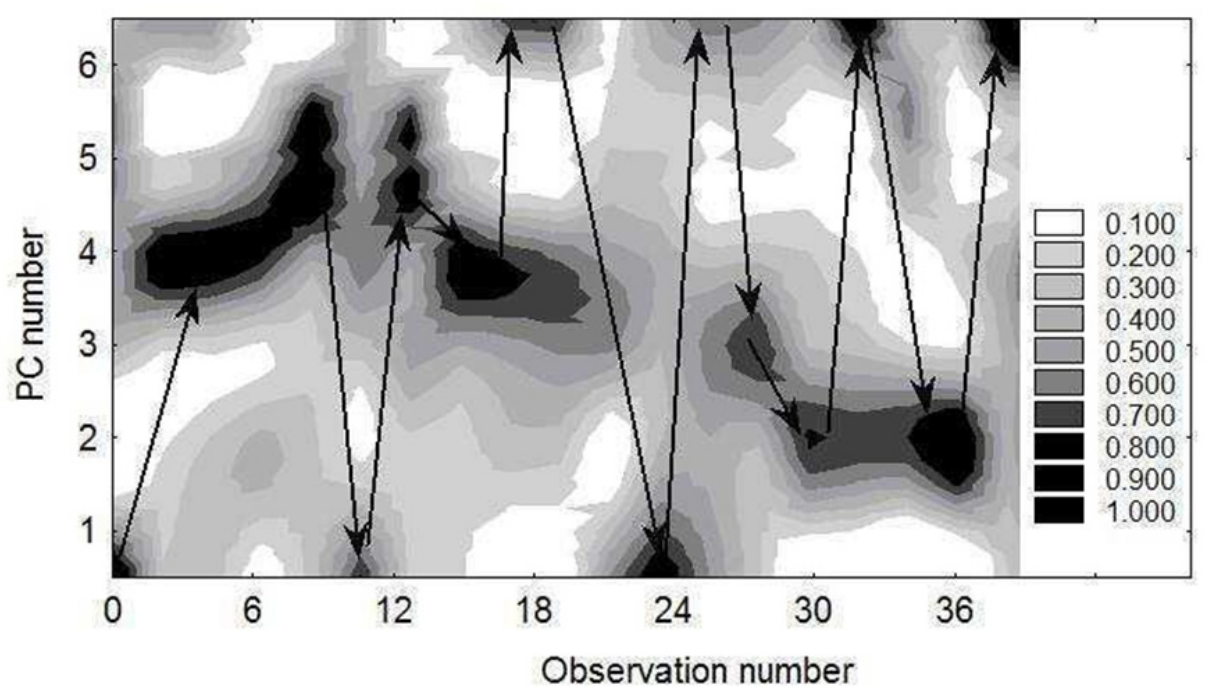

Figure 2. Transient communities of painful zones

Note. The dark regions show high values of projections of zones on given PCs corresponding to larger projection values of vectors defining reported body pain sets. 
Pain distribution patterns by exercise mode (e.g., cycling vs. running) was not shown to affect the total number of changes in pain locations $(\mathrm{Z}=-0.18, \mathrm{p}=.86, \mathrm{~d}=0.11,95 \% \mathrm{CI}$ : $(-6.16,5.21))$ and pain stability $(\mathrm{Z}=-1.78, \mathrm{p}=.07$, $\mathrm{d}=0.56,95 \% \mathrm{CI}:(-33.4,21.93))$.

The pain distribution patterns significantly affected the total number of changes in pain locations $F(2,19)=$ 4.03; $\mathrm{p}<.04$. Tukey post hoc comparisons resulted in a significant difference between adders' and jumpers' patterns, $\mathrm{p}<.035, \mathrm{~d}=1.88,95 \% \mathrm{CI}:(-2.84,3.39))$. Adders, jumpers, and adders-jumpers, respectively, reported 6 $\pm 2.45,21.25 \pm 11.35$ and $16.08 \pm 7.61$ changes (see Figure 3).

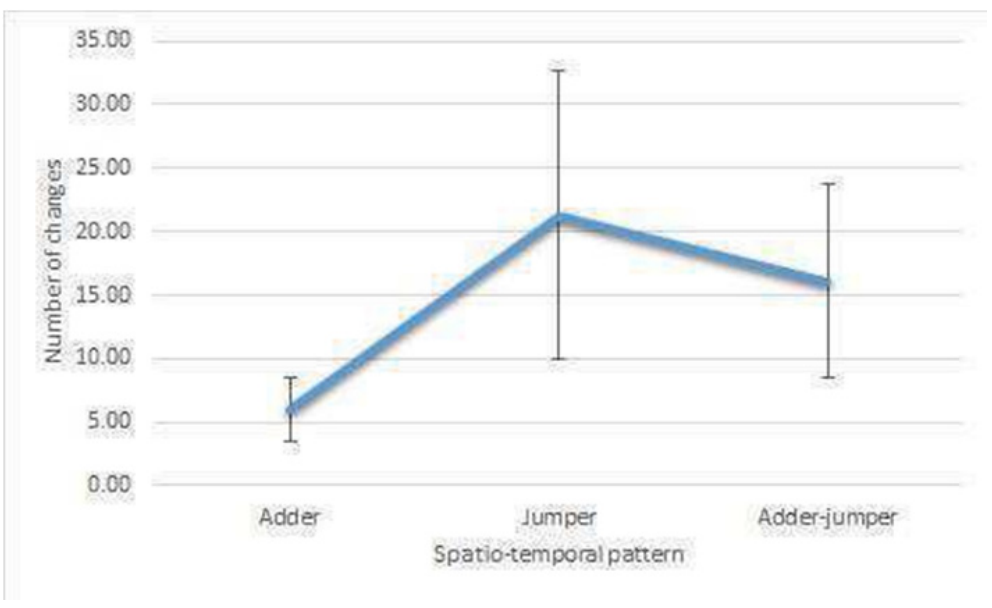

Figure 3. Changes in pain locations

Note. The total number of changes in pain locations within different spatio- temporal patterns. Data are presents as mean $\pm \mathrm{SD}$.

\subsection{Pain Stability}

Pain distribution patterns affected pain stability (i.e., time spent within the same pain location/s), $F(2,19)=$ $10.81 ; \mathrm{p}<.001$. Adders, jumpers, and adders-jumpers, respectively, remained stable in their pattern for an average duration of $143.45 \mathrm{~s} \pm 59.7,51.78 \mathrm{~s} \pm 10.11$, and $62.28 \mathrm{~s} \pm 25.11$, respectively (see Figure 4). Results from Tukey post analysis suggested significant differences between adders and jumpers $\mathrm{p}<.003, \mathrm{~d}=2.26,95 \% \mathrm{CI}$ : $(-34.74,8.52)$; specifically between adders and adder-jumpers patterns $\mathrm{p}<.001, \mathrm{~d}=2.24,95 \% \mathrm{CI}:(-34.76,17.8)$. Finally, task endurance was associated with total amount of changes in pain locations $(r=.46, p=.04)$.

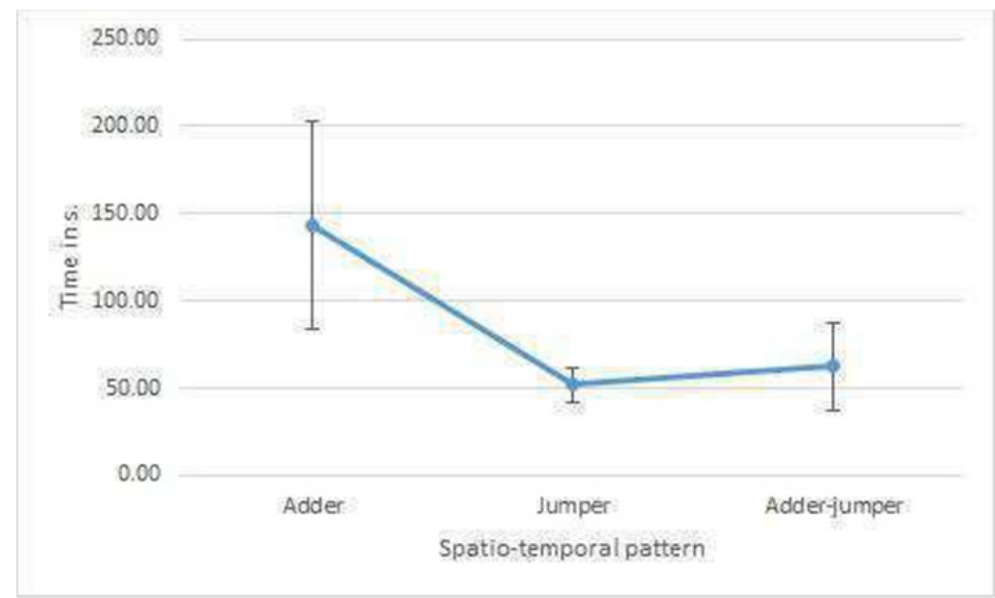

Figure 4. Pain stability

Note. The time spent within the same pain location within different spatio- temporal patterns. Data are presents as mean $\pm \mathrm{SD}$. 


\subsection{Symmetric Pain}

Results also indicated that the symmetric pain (i.e., the type of axial distribution of the same locations in right and left sides of the body) reached its highest values in the mid-point (73.8\% of pain was symmetric) of cycling and at the end (70.7\%) of running (see Figure 5). The Friedman ANOVA revealed a significant effect of time on number of symmetric pain locations $\chi^{2}(10,4)=16.17 \mathrm{p}=.003$ in running, but not in cycling $\chi^{2}(10,4)=7.7, p=.1$. Cohen's $\mathrm{d}$ values between the temporal windows corresponded to: 1 st and 5 th windows $(\mathrm{p}=.01 ; \mathrm{d}=1.13,95 \% \mathrm{CI}:(0.23$, $2.02))$, 2 nd and 4 th windows $(p=.03 ; d=0.62,95 \% \mathrm{CI}:(0.33,1.37)), 3$ nd and 5 th windows $(p=.02 ; d=0.78,95 \%$ CI:( $0.2,1.52))$ in running.

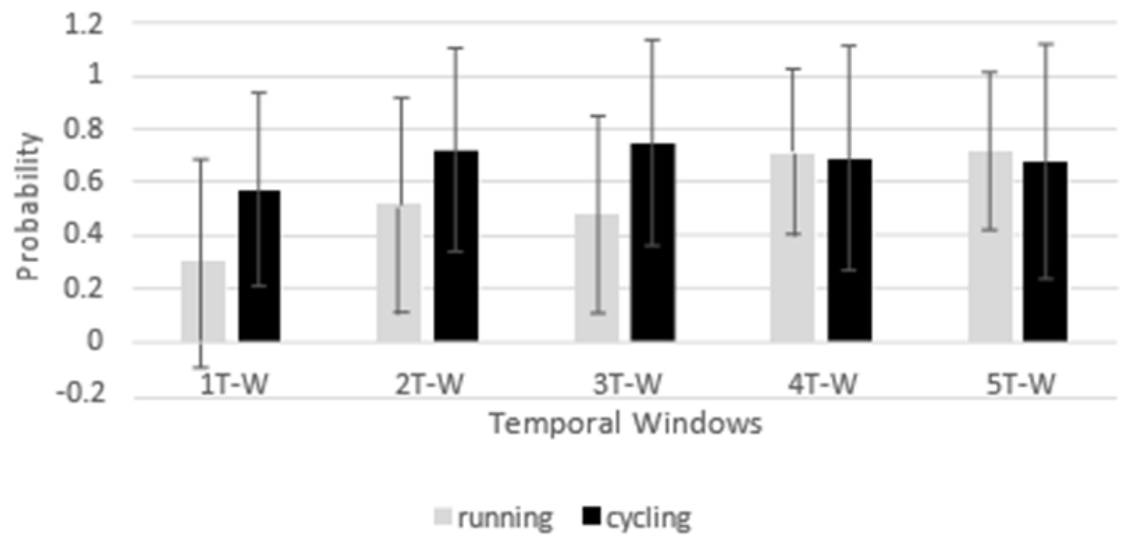

Figure 5. Probability of symmetric pain

Note. The group pulled probabilities of locations with pain during cycling and running tasks in 5 temporal windows in a given sample $(n=10)$. Data are presents as mean \pm SD.

\section{Discussion}

The purpose of this study was to investigate the dynamical distribution of pain in constant cycling and running tasks up to the point of exhaustion. Our main findings revealed three idiosyncratic pain distribution patterns during both cycling and running tasks: (1) adders, (2) jumpers and, (3) adder-jumpers. These idiosyncratic pain distribution patterns were identified in both tasks even though the specific pain locations differed within and between the tasks. These results are partly consistent with previous ones attesting to the importance of identification of individual movement patterns (Schöllhorn, 2003) and idiosyncratic pain differences (Wiech, Ploner, \& Tracey, 2008).

Specifically, participants in the present study presented idiosyncratic differences in the amount of changes they reported with regards to their pain locations (i.e., jumper vs. adder). Importantly also, increased reports of changes in perceived painful locations were associated with increased task endurance. To that end, jumper pattern for instance seemed to have a positive influence on task endurance.

In contrast to the group pulled data, evaluation of the individual idiosyncratic data/dynamics within the present framework did not only help distinguish idiosyncratic pain distribution patterns, but also revealed that the number of painful locations may not increase with time spent on task. Specifically, some participants (i.e., jumpers) did not increase the number of painful locations. This is important to consider in that jumpers may have experienced less pain intensity throughout the task as they have not added novel painful locations to the ones they initially reported. Drawing upon these results, it is conceivable that the pain distribution patterns during running and cycling may have exerted some effects on the accumulated effort. All in all, we observed that throughout the tasks, participants switched their attention to painful locations in a spontaneous manner. However, nearing exhaustion the growth in the number of painful locations became evident.

With regards to a novel taxonomy of pain, the literature includes several previous attempts related to complex spatial pain patterns (Schmidt \& Baymeister, 2007). This said, there is a consensus that although the focus on individual body sites matters, greater attention should be devoted to the examination of the multisite pain (Leveille et al., 2007). The later in part explains the use of the 19 multisite location scale in clinical practice (Wolfe et al., 2011), as well as the use of the 50-item pain scale in the current framework - to allow a more precise measurement 
of the newly arising sites throughout the exhausting exercise. Within the exercise science, no established taxonomy exists for specific pain distribution patterns and, to our knowledge, this is the first attempt to define idiosyncratic pain distribution patterns during exercise. From a measurement standpoint, it is also important to note that although the perception of pain is typically localized within the body, pain is a highly subjective experience (Coghill, McHaffie, \& Yen, 2003). The subjectivity of a painful experience is not, however, mutually exclusive with its objective quantification, and (subjective observation of pain) remains one of its most reliable indicator. This said, idiosyncratic pain within same locations could depict pain groups with unique and different properties (e.g., back pain as a single site has only a prevalence of $5 \%$ of the cases). Within the current study the use of the 50 -item pain scale together with the dynamic approach facilitated a focus on the idiosyncratic data and helped delineate idiosyncratic pain distribution patterns.

Considered together, these results suggest that the model of exhaustive exercises seems to attest to the changing dynamics of signaling processes of pain. To that end, an interesting follow up could include the exploration of potential similarities between the spatiotemporal distributions of exertive pain and select chronic pain presentations. In the case of Fibromyalgia (FM) for instance, the chronic pain distributes within an axial distribution in the upper and lower quadrants, and the right and left sides of the body (Wolfe et al., 1990). In further similarity to chronic pain presentations, the present data suggested a symmetric distribution of pain locations independently of the idiosyncratic distribution pattern. Specifically herein, we have not only identified a multisite pain location phenomenon but also revealed a symmetrical distribution of pain in the right and left sides of the body. Consequently, further investigation of these potential common grounds shared between exercise-related pain and chronic pain presentations can further deepen the understanding of the pain experience in general.

In summary, present results seem to suggest two different patterns of pain-attention dynamics in cycling and running tasks: a rigid pattern of shifting between previously reported pain locations, and a more flexible pattern of shifting to new pain locations. These results suggest that the model of exhaustive exercise is most likely characterized by the changing dynamics of signaling processes of pain.

The potential contributions of the present study come in three folds. First, this study provides a novel framework for examining the dynamics of topologically defined zones of body pain during constant power exercise, and thereby further adds to the understanding of pain perception in general. Second, in the absence of a currently established taxonomy, the results from this study help advance an initial taxonomy for pain distribution patterns: adder, jumper and adder-jumper. Third, the present results reveal an association between exercise endurance and the number of switches among painful locations during exercise. This finding is particularly relevant for the realm of performance science where task endurance is a central topic of interest. Future research is warranted to test the potential link between the idiosyncratic fitness levels and the reported pain distribution patterns. Specifically, it could be beneficial to investigate whether highly trained individuals would present particular distribution patterns over less trained individuals. This tentative assumption is drawn from the current results that suggest that the total number of reported changes in painful locations is related to task endurance. To that end, jumper could possibly be the prevailing pattern among highly trained individuals and delineating such linkages could prove useful.

Within applied realms, practitioners could make use of these findings for developing a better understanding of exercise-induced pain. It is known that heightened somatic pain causes negative affective responses and precludes one from exercise adherence (Zenko, Ekkekakis, \& Ariely, 2016). Consequently, practitioners would benefit from further grasping the phenomenon of pain and developing individualized management strategies for exercisers depending on their idiosyncratic pain distribution patterns. For instance, early identification of an exerciser's idiosyncratic pain distribution pattern as an adder can help interventions to curtail the overload that individual can place upon their system by constantly perceiving novel painful locations during exercise. As such for an adder, perhaps the use of a dissociative strategy such an external imagery (Razon et al., 2010), listening to music (Chow \& Etnier, 2017) and/or alternative attention modulation strategies that turn one's attention outwards could reduce exertion and potentially help prolong task adherence. Similarly, an adder-jumper that adds and jumps in between locations could be trained to keep a more singular focus of attention for optimal performance - since they could otherwise be relatively prone to experiencing distraction as a result of constantly alternating attentional focus.

Two potential limitations are observed in the present study. First, in thiss study neither the intensity nor the quality of pain was measured; hence, we cannot confirm that pain intensity was higher in adders than in jumpers. Second, the idiosyncratic expectations about the painful locations were not evaluated. This is important in that expectations of pain are known to alter the strength of spinal nociceptive responses in humans (Pfaff, 2013). As such, participants could have perceived painful what they initially expected to be painful.

As a word of conclusion, this study remains pioneer in delineating the idiosyncratic distribution patterns of pain 
during exhaustive exercise. While more research is needed to expand on these findings, exercise practitioners could make use of these results to optimize pain management and performance outcomes in exercise settings allowing optimal performance and prolonged adherence to effort. Additionally, medical professionals may find value in the potential translations of this preliminary results toward a more effective and efficient management of an otherwise particularly complex phenomenon such as pain (Williams \& Craig, 2016).

\section{Acknowledgements}

This study was supported by the Institut Nacional d'Educació Física de Catalunya (INEFC), the Generalitat de Catalunya. Dr. Agnè Slapšinskaitė was the recipient of a predoctoral fellowship from the Institut Nacional d'Educació Física de Catalunya (INEFC).

\section{Ethical Issues}

Participants completed an informed consent form. All the experimental procedures were approved by the Clinical Research Ethics Committee of the Sports Administration of Catalonia and were carried out according to the Helsinki Declaration. Registration: ClinicalTrials.gov ID: NCT02876705

\section{Competing Interests Statement}

The authors declare that there are no competing or potential conflicts of interest.

\section{References}

Astokorki, A. H. Y., \& Mauger, A. R. (2017). Tolerance of exercise - induced pain at a fixed rating of perceived exertion predicts time trial cycling performance. Scandinavian Journal of Medicine \& Science in Sports, 27(3), 309-317. https://doi.org/10.1111/sms.12659

Balagué, N., Hristovski, R., Garcia, S., Aragonés, D., Razon, S., \& Tenenbaum, G. (2015). Intentional thought dynamics during exercise performed until volitional exhaustion. Journal of Sports Sciences, 33(1), 48-57. https://doi.org/10.1080/02640414.2014.921833

Borg, G. A. (1998). Borg's perceived exertion and pain scales. Champaign, Illinois, IL: Human Kinetics.

Chalmers, D. J. (1996). The conscious mind: In search of a fundamental theory. Oxford University Press.

Coghill, R. C., McHaffie, J. G., \& Yen, Y.-F. (2003). Neural correlates of interindividual differences in the subjective experience of pain. Proceedings of the National Academy of Sciences of the United States of America, 100(14), 8538-8542. https://doi.org/10.1073/pnas.1430684100

Cook, D. B., O'Connor, P. J., Oliver, S. E., \& Lee, Y. (1998). Sex differences in naturally occurring leg muscle pain and exertion during maximal cycle ergometry. The International Journal of Neuroscience, 95(3-4), 183-202. https://doi.org/10.3109/00207459809003340

Dannecker, E. A., \& Koltyn, K. F. (2014). Pain during and within hours after exercise in healthy adults. Sports Medicine, 44(7), 921-942. https://doi.org/10.1007/s40279-014-0172-z

Faul, F., Erdfelder, E., Lang, A.-G., \& Buchner, A. (2007). G*Power 3: A flexible statistical power analysis program for the social, behavioral, and biomedical sciences. Behavior Research Methods, 39(2), 175-191. https://doi.org/10.3758/BF03193146

Graven-Nielsen, T., \& Arendt-Nielsen, L. (2010). Assessment of mechanisms in localized and widespread musculoskeletal pain. Nat Rev Rheumatol, 6(10), 599-606. Retrieved from http://dx.doi.org/10.1038/nrrheum.2010.107

Haile, L., Gallagher Jr., M., \& J. Robertson, R. (2015). Exercise-Induced Muscle Pain. In Perceived Exertion Laboratory Manual SE - 3 (pp. 21-27). Springer New York. https://doi.org/10.1007/978-1-4939-1917-8_3

Leveille, S. G., Bean, J., Ngo, L., McMullen, W., \& Guralnik, J. M. (2007). The pathway from musculoskeletal pain to mobility difficulty in older disabled women. Pain, 128(1), 69-77.

Margolis, R. B., Tait, R. C., \& Krause, S. J. (1986). A rating system for use with patient pain drawings. Pain, 24(1), $57-65$.

Pfaff, D. W. (2013). Neuroscience in the 21st century: From basic to clinical. Neuroscience in the 21st Century: From Basic to Clinical. https://doi.org/10.1007/978-1-4614-1997-6

Rabinovich, M. I., Tristan, I., \& Varona, P. (2015). Hierarchical nonlinear dynamics of human attention. Neuroscience and Biobehavioral Reviews, 55, 18-35. https://doi.org/10.1016/j.neubiorev.2015.04.001

Razon, S., Basevitch, I., Filho, E., Land, W., Thompson, B., Biermann, M., \& Tenenbaum, G. (2010). Associative 
and Dissociative Imagery Effects on Perceived Exertion and Task Duration. Journal of Imagery Research in Sport and Physical Activity, 5(1). https://doi.org/10.2202/1932-0191.1044

Schmidt, C. O., \& Baymeister, S. E. (2007). Simple patterns behind complex spatial pain reporting? Assessing a classification of multisite pain reporting in the general population. PAIN®, 113(1-3), 174-182. https://doi.org/10.1016/j.pain.2007.04.022

Schöllhorn, W. (2003). Coordination dynamics and its consequences on sports. International Journal of Computer Science in Sport, 2(2), 40-46.

Slapsinskaite, A., Hristovski, R., Razon, S., Balagué, N., \& Tenenbaum, G. (2017). Metastable Pain-Attention Dynamics during Incremental Exhaustive Exercise, 7(January), 1-9. https://doi.org/10.3389/fpsyg.2016.02054

Slapsinskaite, A., Razon, S., Balagué Serre, N., Hristovski, R., \& Tenenbaum, G. (2015). Local pain dynamics during constant exhaustive exercise. Plos One, 10(9), e0137895. https://doi.org/10.1371/journal.pone.0137895

Tarr, J., \& Thomas, H. (2011). Mapping embodiment: methodologies for representing pain and injury. Qualitative Research, 11(2), 141-157. https://doi.org/10.1177/1468794110394067

Tenenbaum, G., Fogarty, G., Stewart, E., Calcagnini, N., Kirker, B., Thorne, G., \& Christensen, S. (1999). Perceived discomfort in running: Scale development and theoretical considerations. Journal of Sports Sciences, 17(3), 183-196. https://doi.org/10.1080/026404199366082

Tripathi, L., \& Kumar, P. (2014). Challenges in pain assessment: Pain intensity scales. Indian Journal of Pain, 28(2), 61-70. https://doi.org/10.4103/0970-5333.132841

Villemure, C., \& Bushnell, M. C. (2002). Cognitive modulation of pain: How do attention and emotion influence pain processing? Pain, 95(3), 195-199. https://doi.org/10.1016/S0304-3959(02)00007-6

Wiech, K., Ploner, M., \& Tracey, I. (2008). Neurocognitive aspects of pain perception. Trends in Cognitive Sciences, 12(July), 306-313. https://doi.org/10.1016/j.tics.2008.05.005

Williams, A. C., \& Craig, K. D. (2016). Updating the definition of pain. Pain. https://doi.org/10.1097/j.pain.0000000000000613

Wolfe, F., Clauw, D. J., Fitzcharles, M. A., Goldenberg, D. L., Häuser, W., Katz, R. S., ... Winfield, J. B. (2011). Fibromyalgia criteria and severity scales for clinical and epidemiological studies: a modification of the ACR Preliminary Diagnostic Criteria for Fibromyalgia. The Journal of Rheumatology, 38(6), 1113-1122. https://doi.org/10.3899/jrheum.100594

Wolfe, F., Smythe, H. A., Yunus, M. B., Bennett, R. M., Bombardier, C., Goldenberg, D. L., ... Clark, P. (1990). The American College of Rheumatology 1990 criteria for the classification of fibromyalgia. Arthritis \& Rheumatism, 33(2), 160-172. https://doi.org/10.3899/jrheum.100594

Yeomans, K., A., \& Golder, P., A. (1982). The Guttman-Kaiser criterion as a predictor of the number of common factors. Journal of the Royal Statistical Society: Series B, 3, 221-229.

\section{Copyrights}

Copyright for this article is retained by the author(s), with first publication rights granted to the journal.

This is an open-access article distributed under the terms and conditions of the Creative Commons Attribution license (http://creativecommons.org/licenses/by/4.0/). 